\title{
Symmetry and regularity of positive solutions to integral systems with Bessel potential
}

\section{Xiaoli Chen ${ }^{*}$ and Changsen Wang}

"Correspondence:

littleli_chen@163.com

Department of Mathematics, Jiangxi

Normal University, Nanchang,

Jiangxi 330022, P.R. China

\begin{abstract}
In this paper, we are concerned with the symmetry and regularity of positive solutions of the following integral system: $u(x)=\int_{\mathbb{R}^{n}} \frac{G_{\alpha}(x-y) w^{\prime}(y) v^{q}(y)}{|y|^{\beta}} d y$,

$v(x)=\int_{\mathbb{R}^{n}} \frac{G_{\alpha}(x-y) u^{p}(y) w^{r}(y)}{\left.|y|\right|^{\beta}} d y, w(x)=\int_{\mathbb{R}^{n}} \frac{G_{\alpha}(x-y) u^{p}(y) v^{q}(y)}{|y|^{\beta}} d y$, where $G_{\alpha}(x)$ is the $\alpha$ th-order Bessel kernel, $n \geq 3,0 \leq \beta<\alpha<n, 1<p, q, r<\frac{n-\beta}{\beta}$ and $\frac{1}{p+1}+\frac{1}{q+1}+\frac{1}{r+1}>\frac{2 n-\alpha+\beta}{n}$. We show that every positive solution triple $(u, v, w)$ of the system is radially symmetric and monotonic decreasing about some point by the moving planes method in integral forms. Moreover, by the regularity lifting method, we prove that $(u, v, w)$ belongs to $L^{\infty}\left(\mathbb{R}^{n}\right) \times L^{\infty}\left(\mathbb{R}^{n}\right) \times L^{\infty}\left(\mathbb{R}^{n}\right)$ and which is then locally Hölder continuous.

MSC: $45 \mathrm{E} 10 ; 45 \mathrm{G} 05$
\end{abstract}

Keywords: radial symmetry; $L^{\infty}$ bounds; local Hölder continuous

\section{Introduction}

In this paper, we are concerned with the symmetry and regularity of positive solutions of the following integral system:

$$
\left\{\begin{array}{l}
u(x)=\int_{\mathbb{R}^{n}} \frac{G_{\alpha}(x-y) w^{r}(y) v^{q}(y)}{\mid y^{\beta}} d y, \\
v(x)=\int_{\mathbb{R}^{n}} \frac{G_{\alpha}(x-y) u^{p}(y) w^{r}(y)}{|y|^{\beta}} d y, \\
w(x)=\int_{\mathbb{R}^{n}} \frac{G_{\alpha}(x-y) u^{p}(y) v^{q}(y)}{|y|^{\beta}} d y,
\end{array}\right.
$$

where $G_{\alpha}(x)$ is the $\alpha$ th-order Bessel kernel, $n \geq 3,0 \leq \beta<\alpha<n, p, q, r>1$, and

$$
\frac{1}{p+1}+\frac{1}{q+1}+\frac{1}{r+1}>\frac{2 n-\alpha+\beta}{n}
$$

Problem (1.1) is related to the $\alpha$ th-order Bessel potentials $B_{\alpha}=(-\Delta+i d)^{-\frac{\alpha}{2}}$. The $\alpha$ thorder Bessel kernel $G_{\alpha}(x)$ is given by

$$
G_{\alpha}(x)=\frac{1}{(4 \pi)^{\frac{\alpha}{2}} \Gamma\left(\frac{\alpha}{2}\right)} \int_{0}^{\infty} \exp \left(-\frac{\pi}{s}|x|^{2}\right) \exp \left(-\frac{s}{4 \pi}\right) s^{\frac{\alpha-n}{2}} \frac{d s}{s} .
$$


Suppose $f \in L^{p}\left(\mathbb{R}^{n}\right)$ with $1 \leq p \leq \infty$, the $\alpha$ th-order Bessel potential $B_{\alpha}=(-\Delta+i d)^{-\frac{\alpha}{2}}$ of $f$ is defined by

$$
B_{\alpha}(f)= \begin{cases}G_{\alpha} * f, & \alpha>0, \\ f, & \alpha=0\end{cases}
$$

where $*$ denotes the convolution of functions.

The symmetry of the solutions to nonlinear elliptic problems are in general investigated by the moving planes method, which was first used by Alexanderoff for differential equations, and developed by Serrin [1], Gidas et al. [2], Caffarelli et al. [3] etc. In particular, it was considered in $[2,4]$ the radial symmetry and monotonicity of nonnegative solutions of nonlinear elliptic equations by the moving planes method. Such a method is based on the maximum principle, and hence it cannot be directly applied to problems in the absence of the maximum principle. Especially for nonlinear integral equations or systems, one needs a replacement of the maximum principle. It was observed by Chen et al. in $[5,6]$ that when studying the radial symmetry and monotonicity of nonnegative solutions of integral equations, one can use a Hardy-Littlewood-Sobolev (HLS) type inequality, instead of the maximum principle, in the moving planes method. They show in $[5,6]$ that nonnegative solutions of integral equations

$$
u(x)=\int_{\mathbb{R}^{n}} \frac{u^{\frac{n+\alpha}{n-\alpha}}(y)}{|x-y|^{n-\alpha}} d y
$$

are radially symmetric. Since then, radial symmetry and monotonicity of positive solutions for integral equations and integral systems with the Riesz potential have been extensively studied; see [5-7] and [8] etc.

To study the properties of positive solutions for integral equations with Bessel kernel, Ma and Chen [9] establish a HLS type inequality for the Bessel potentials and obtain the radial symmetry and monotonicity of positive solutions of integral equations by using the moving planes method in integral forms. Later, they [10] studied the following integral system with Bessel kernel:

$$
u=G_{\alpha_{1}} * v^{q}, \quad v=G_{\alpha_{2}} * u^{p} .
$$

If $\alpha_{1}=\alpha_{2}=1$, this system is the stationary Dirac-Schrödinger system, and if $\alpha_{1}=\alpha_{2}=2$, system (1.4) becomes the stationary Schrödinger system. More equations associated with Bessel potential can be found in [11-14] etc.

Recently, Chen and Yang established in [15] the HLS type inequality for the Bessel potentials with double weights, and they showed that positive solutions pairs $(u, v) \in$ $L^{p+1}\left(\mathbb{R}^{n}\right) \times L^{q+1}\left(\mathbb{R}^{n}\right)$ of the integral system

$$
u=G_{\alpha} * \frac{v^{q}(y)}{|y|^{\beta}}, \quad v=G_{\alpha} * \frac{u^{p}(y)}{|y|^{\beta}}
$$

are Hölder continuous, radially symmetric, and strictly decreasing about the origin. Moreover, in [16], the authors consider the radial symmetry and uniqueness of positive solution 
pairs $(u, v)$ of integral system

$$
u=G_{\alpha} * \frac{u^{p}(y) v^{q}(y)}{|y|^{\beta}}, \quad v=G_{\alpha} * \frac{\nu^{p}(y) u^{q}(y)}{|y|^{\beta}} .
$$

In this paper, we will investigate the regularity and symmetry as well as Hölder continuity of solutions to problem (1.1), which can be deduced to neither problem (1.5) nor (1.6). Firstly, using the moving planes method in integral forms, we have the following symmetric result.

Theorem 1.1 Under condition (1.2), any positive solution triple $(u, v, w) \in L^{p+1}\left(R^{n}\right) \times$ $L^{q+1}\left(R^{n}\right) \times L^{r+1}\left(R^{n}\right)$ to system (1.1) is radially symmetric and monotone decreasing about some point in $\mathbb{R}^{n}$.

Secondly, by the regularity lifting method, we show the boundedness of each component of the solution triple. To state it precisely, we have the following.

Theorem 1.2 Let triple $(u, v, w) \in L^{p+1}\left(\mathbb{R}^{n}\right) \times L^{q+1}\left(\mathbb{R}^{n}\right) \times L^{r+1}\left(\mathbb{R}^{n}\right)$ be a positive solution to integral system (1.1), and $p, q$, r satisfies (1.2). Then $(u, v, w) \in L^{\infty}\left(\mathbb{R}^{n}\right) \times L^{\infty}\left(\mathbb{R}^{n}\right) \times L^{\infty}\left(\mathbb{R}^{n}\right)$.

In the proof of Theorem 1.2, we first lift the integrability of a suitable cut-off function of the solution by the regularity lifting method to some $L^{\tilde{q}}$, and then we show that they are actually in $L^{\infty}$. Finally, we assert that the solution triple is locally Hölder continuous.

Theorem $1.3 u, v$, and $w$ are locally Hölder continuous.

In Section 2, we show that $(u, v, w)$ is radially symmetric by the moving planes method. Then, using the regularity lifting method, we prove $(u, v, w) \in L^{\infty}\left(\mathbb{R}^{n}\right) \times L^{\infty}\left(\mathbb{R}^{n}\right) \times L^{\infty}\left(\mathbb{R}^{n}\right)$ in Section 3. In the last section, we prove Theorem 1.3.

\section{Proof of Theorem 1.1}

This section is devoted to proving the symmetry and monotonicity of positive solutions to system (1.1). Assume that $\lambda \in \mathbb{R}$ is a given real number, we define $T_{\lambda}=\left\{x \in \mathbb{R}^{n} \mid x_{1}=\lambda\right\}$, $\Sigma_{\lambda}=\left\{x=\left(x_{1}, x_{2}, \ldots, x_{n}\right) \in \mathbb{R}^{n} \mid x_{1} \leq \lambda\right\}$. For $x \in \Sigma_{\lambda}$, let $x_{\lambda}=\left(2 \lambda-x_{1}, x_{2}, \ldots, x_{n}\right)$, and define

$$
u_{\lambda}(x)=u\left(x_{\lambda}\right), \quad v_{\lambda}(x)=v\left(x_{\lambda}\right), \quad w_{\lambda}(x)=w\left(x_{\lambda}\right) .
$$

Lemma 2.1 For any positive solution $(u, v, w)$ of (1.1), we have

$$
\begin{aligned}
& u(x)-u_{\lambda}(x)=\int_{\Sigma_{\lambda}}\left(G_{\alpha}(x-y)-G_{\alpha}\left(x_{\lambda}-y\right)\right)\left(\frac{v^{q} w^{r}}{|y|^{\beta}}-\frac{v_{\lambda}^{q} w_{\lambda}^{r}}{\left|y_{\lambda}\right|^{\beta}}\right) d y, \\
& v(x)-v_{\lambda}(x)=\int_{\Sigma_{\lambda}}\left(G_{\alpha}(x-y)-G_{\alpha}\left(x_{\lambda}-y\right)\right)\left(\frac{u^{p} w^{r}}{|y|^{\beta}}-\frac{u_{\lambda}^{p} w_{\lambda}^{r}}{\left|y_{\lambda}\right|^{\beta}}\right) d y, \\
& w(x)-w_{\lambda}(x)=\int_{\Sigma_{\lambda}}\left(G_{\alpha}(x-y)-G_{\alpha}\left(x_{\lambda}-y\right)\right)\left(\frac{\nu^{q} u^{p}}{|y|^{\beta}}-\frac{v_{\lambda}^{q} u_{\lambda}^{p}}{\left|y_{\lambda}\right|^{\beta}}\right) d y .
\end{aligned}
$$


Proof Let $\Sigma_{\lambda}^{c}=\left\{x=\left(x_{1}, x_{2}, \ldots, x_{n}\right) \in \mathbb{R}^{n} \mid x_{1}>\lambda\right\}$. Since $\left|x-y_{\lambda}\right|=\left|x_{\lambda}-y\right|,|x-y|=\left|x_{\lambda}-y_{\lambda}\right|$, and $G_{\alpha}$ is radially symmetric in $\mathbb{R}^{n}$, it follows from (1.1) that

$$
\begin{aligned}
u(x) & =\int_{\Sigma_{\lambda}} \frac{G_{\alpha}(x-y) w^{r}(y) v^{q}(y)}{|y|^{\beta}} d y+\int_{\Sigma_{\lambda}^{c}} \frac{G_{\alpha}(x-y) w^{r}(y) v^{q}(y)}{|y|^{\beta}} d y \\
& =\int_{\Sigma_{\lambda}} \frac{G_{\alpha}(x-y) w^{r}(y) v^{q}(y)}{|y|^{\beta}} d y+\int_{\Sigma_{\lambda}} \frac{G_{\alpha}\left(x-y_{\lambda}\right) w_{\lambda}^{r}(y) v_{\lambda}^{q}(y)}{\left|y_{\lambda}\right|^{\beta}} d y \\
& =\int_{\Sigma_{\lambda}} \frac{G_{\alpha}(x-y) w^{r}(y) v^{q}(y)}{|y|^{\beta}} d y+\int_{\Sigma_{\lambda}} \frac{G_{\alpha}\left(x_{\lambda}-y\right) w_{\lambda}^{r}(y) v_{\lambda}^{q}(y)}{\left|y_{\lambda}\right|^{\beta}} d y .
\end{aligned}
$$

Substituting $x$ by $x_{\lambda}$, we obtain

$$
u_{\lambda}(x)=\int_{\Sigma_{\lambda}} \frac{G_{\alpha}\left(x_{\lambda}-y\right) w^{r}(y) v^{q}(y)}{|y|^{\beta}} d y+\int_{\Sigma_{\lambda}} \frac{G_{\alpha}(x-y) w_{\lambda}^{r}(y) v_{\lambda}^{q}(y)}{\left|y_{\lambda}\right|^{\beta}} d y .
$$

Hence,

$$
u(x)-u_{\lambda}(x)=\int_{\Sigma_{\lambda}}\left(G_{\alpha}(x-y)-G_{\alpha}\left(x_{\lambda}-y\right)\right)\left(\frac{\nu^{q}(y) w^{r}(y)}{|y|^{\beta}}-\frac{v_{\lambda}^{q}(y) w_{\lambda}^{r}(y)}{\left|y_{\lambda}\right|^{\beta}}\right) d y
$$

Similarly, we have (2.2) and (2.3). The proof is complete.

To prove Theorem 1.1, we need Hardy-Littlewood-Sobolev's inequality for the Bessel potential, which can be found in [15].

Lemma 2.2 Let $0 \leq \alpha<n, 1<l, d<\frac{n}{\alpha}, \tau, \beta \geq 0$. In addition $n\left(1-\frac{1}{l}-\frac{1}{d}+\frac{\alpha}{n}\right)>\beta+\tau>n(1-$ $\left.\frac{1}{l}-\frac{1}{d}\right)$. Then there exists a positive constant $C$ independent of $f \in L^{l}\left(\mathbb{R}^{n}\right)$ and $h \in L^{d}\left(\mathbb{R}^{n}\right)$ such that the following inequality holds:

$$
\left|\int_{\mathbb{R}^{n}} \int_{\mathbb{R}^{n}} \frac{f(x) G_{\alpha}(x-y) h(y)}{|x|^{\tau}|y|^{\beta}} d x d y\right| \leq C\|f\|_{l}\|h\|_{d} .
$$

Furthermore, let

$$
\operatorname{Th}(x)=\int_{\mathbb{R}^{n}} \frac{G_{\alpha}(x-y) h(y)}{|x|^{\tau}|y|^{\beta}} d y,
$$

then

$$
\|T h\|_{l^{\prime}}=\sup _{\|f\|_{l}=1}|\langle T h, f\rangle| \leq C\|h\|_{d}
$$

where $\frac{1}{l}+\frac{1}{l^{\prime}}=1,1+\frac{1}{l^{\prime}} \geq \frac{1}{d}+\frac{n-\alpha+\beta+\tau}{n}, h \in L^{d}\left(\mathbb{R}^{n}\right)$, which means $d>\frac{n l^{\prime}}{n+(\alpha-\beta-\tau)}$.

Proof of Theorem 1.1 First, we show that there exists a negative number $\lambda$, such that

$$
u(x) \leq u_{\lambda}(x), \quad v(x) \leq v_{\lambda}(x), \quad w(x) \leq w_{\lambda}(x), \quad \forall x \in \Sigma_{\lambda}
$$

Define

$$
\Sigma_{\lambda}^{u}=\left\{x \in \Sigma_{\lambda} \mid u(x)>u_{\lambda}(x)\right\}, \quad \Sigma_{\lambda}^{v}=\left\{x \in \Sigma_{\lambda} \mid v(x)>v_{\lambda}(x)\right\}
$$


and

$$
\Sigma_{\lambda}^{w}=\left\{x \in \Sigma_{\lambda} \mid w(x)>w_{\lambda}(x)\right\}
$$

We derive from Lemma 2.1 that

$$
u(x)-u_{\lambda}(x)=\int_{\Sigma_{\lambda}}\left(G_{\alpha}(x-y)-G_{\alpha}\left(x_{\lambda}-y\right)\right)\left(\frac{\nu^{q}(y) w^{r}(y)}{|y|^{\beta}}-\frac{v_{\lambda}^{q}(y) w_{\lambda}^{r}(y)}{\left|y_{\lambda}\right|^{\beta}}\right) d y .
$$

Observing that for $x, y \in \Sigma_{\lambda},|x-y| \leq\left|x_{\lambda}-y\right|,|y| \geq\left|y_{\lambda}\right|$, and that $G_{\alpha}$ is decreasing, if $x \in \Sigma_{\lambda}^{u}$, we obtain

$$
\begin{aligned}
0 \leq & u(x)-u_{\lambda}(x) \\
\leq & \int_{\Sigma_{\lambda}}\left(G_{\alpha}(x-y)-G_{\alpha}\left(x_{\lambda}-y\right)\right)\left(\frac{w^{r}\left(v^{q}-v_{\lambda}^{q}\right)}{|y|^{\beta}}+\frac{v_{\lambda}^{q}\left(w^{r}-w_{\lambda}^{r}\right)}{|y|^{\beta}}\right) d y \\
= & \int_{\Sigma_{\lambda}^{v}} \frac{G_{\alpha}(x-y) w^{r}\left(v^{q}-v_{\lambda}^{q}\right)}{|y|^{\beta}} d y+\int_{\Sigma_{\lambda}^{w}} \frac{G_{\alpha}(x-y) v_{\lambda}^{q}\left(w^{r}-w_{\lambda}^{r}\right)(y)}{|y|^{\beta}} d y \\
& +\int_{\left(\Sigma_{\lambda}^{v}\right)^{c}}\left(G_{\alpha}(x-y)-G_{\alpha}\left(x_{\lambda}-y\right)\right) \frac{w^{r}\left(v^{q}-v_{\lambda}^{q}\right)(y)}{|y|^{\beta}} d y \\
& +\int_{\left(\Sigma_{\lambda}^{w}\right)^{c}}\left(G_{\alpha}(x-y)-G_{\alpha}\left(x_{\lambda}-y\right)\right) \frac{v_{\lambda}^{q}\left(w^{r}-w_{\lambda}^{r}\right)(y)}{|y|^{\beta}} d y \\
& -\int_{\Sigma_{\lambda}^{v}} G_{\alpha}\left(x_{\lambda}-y\right) \frac{w^{r}\left(v^{q}-v_{\lambda}^{q}\right)(y)}{|y|^{\beta}} d y-\int_{\Sigma_{\lambda}^{w}} G_{\alpha}\left(x_{\lambda}-y\right) \frac{v_{\lambda}^{q}\left(w^{r}-w_{\lambda}^{r}\right)(y)}{|y|^{\beta}} d y \\
\leq & \int_{\Sigma_{\lambda}^{v}} G_{\alpha}(x-y) \frac{w^{r}\left(v^{q}-v_{\lambda}^{q}\right)(y)}{|y|^{\beta}} d y+\int_{\Sigma_{\lambda}^{w}} G_{\alpha}(x-y) \frac{v_{\lambda}^{q}\left(w^{r}-w_{\lambda}^{r}\right)(y)}{|y|^{\beta}} d y \\
\leq & q \int_{\Sigma_{\lambda}^{v}} \frac{G_{\alpha}(x-y) v^{q-1}\left(v-v_{\lambda}\right) w^{r}(y)}{|y|^{\beta}} d y+r \int_{\Sigma_{\lambda}^{w}} \frac{G_{\alpha}(x-y) w^{r-1}\left(w-w_{\lambda}\right) v_{\lambda}^{q}}{|y|^{\beta}} d y \\
= & A(x)+B(x) .
\end{aligned}
$$

Since condition (1.2) holds, we can choose $\frac{1}{d_{1}}=\frac{r}{r+1}+\frac{q-1}{q+1}+\frac{1}{q+1}$. By Hardy-LittlewoodSobolev's inequality and Hölder's inequality, we have

$$
\|A(x)\|_{L^{p+1}\left(\Sigma_{\lambda}^{u}\right)} \leq C\left\|v^{q-1}\left(v-v_{\lambda}\right) w^{r}\right\|_{L^{d_{1}\left(\Sigma_{\lambda}^{v}\right)}} \leq C\|v\|_{L^{q+1}\left(\Sigma_{\lambda}^{v}\right)}^{q-1}\left\|v-v_{\lambda}\right\|_{L^{q+1}\left(\Sigma_{\lambda}^{v}\right)}\|w\|_{L^{r+1}\left(\Sigma_{\lambda}^{\nu}\right)}^{r} .
$$

Similarly, choosing $\frac{1}{d_{2}}=\frac{r-1}{r+1}+\frac{1}{r+1}+\frac{q}{q+1}$, we get

$$
\|B(x)\|_{L^{p+1}\left(\Sigma_{\lambda}^{u}\right)} \leq C\left\|w^{r-1}\left(w-w_{\lambda}\right) v_{\lambda}^{q}\right\|_{L^{d_{2}\left(\Sigma_{\lambda}^{w}\right)}} \leq C\|w\|_{L^{r+1}\left(\Sigma_{\lambda}^{w}\right)}^{r-1}\left\|w-w_{\lambda}\right\|_{L^{r+1}\left(\Sigma_{\lambda}^{w}\right)}\left\|v_{\lambda}\right\|_{L^{q+1}\left(\Sigma_{\lambda}^{v}\right)}^{q} .
$$

Hence,

$$
\begin{aligned}
\left\|u-u_{\lambda}\right\|_{L^{p+1}\left(\Sigma_{\lambda}^{u}\right) \leq} & C\|v\|_{L^{q+1}\left(\Sigma_{\lambda}^{v}\right)}^{q-1}\left\|v-v_{\lambda}\right\|_{L^{q+1}\left(\Sigma_{\lambda}^{v}\right)}\|w\|_{L^{r+1}\left(\Sigma_{\lambda}^{v}\right)}^{r} \\
& +C\|w\|_{L^{r+1}\left(\Sigma_{\lambda}^{w}\right)}^{r-1}\left\|w-w_{\lambda}\right\|_{L^{r+1}\left(\Sigma_{\lambda}^{w}\right)}\left\|v_{\lambda}\right\|_{L^{q+1}\left(\Sigma_{\lambda}^{v}\right)}^{q} .
\end{aligned}
$$


On the other hand, using the fact that $(u, v, w) \in L^{p+1}\left(R^{n}\right) \times L^{q+1}\left(R^{n}\right) \times L^{r+1}\left(R^{n}\right)$, we can choose $\lambda$ small enough, such that

$$
C\|w\|_{L^{r+1}\left(\Sigma_{\lambda}^{\nu}\right)}^{r}\|v\|_{L^{q+1}\left(\Sigma_{\lambda}^{\nu}\right)}^{q-1} \leq \frac{1}{4}, \quad C\|w\|_{L^{r+1}\left(\Sigma_{\lambda}^{w}\right)}^{r}\left\|v_{\lambda}\right\|_{L^{q+1}\left(\Sigma_{\lambda}^{w}\right)}^{q} \leq \frac{1}{4} .
$$

Thus,

$$
\left\|u-u_{\lambda}\right\|_{L^{p+1}\left(\Sigma_{\lambda}^{u}\right)} \leq \frac{1}{4}\left(\left\|v-v_{\lambda}\right\|_{L^{q+1}\left(\Sigma_{\lambda}^{v}\right)}+\left\|w-w_{\lambda}\right\|_{L^{r+1}\left(\Sigma_{\lambda}^{w}\right)}\right) .
$$

Similarly,

$$
\left\|v-v_{\lambda}\right\|_{L^{q+1}\left(\Sigma_{\lambda}^{\nu}\right)} \leq \frac{1}{4}\left(\left\|w-w_{\lambda}\right\|_{L^{r+1}\left(\Sigma_{\lambda}^{w}\right)}+\left\|u-u_{\lambda}\right\|_{L^{p+1}\left(\Sigma_{\lambda}^{u}\right)}\right)
$$

and

$$
\left\|w-w_{\lambda}\right\|_{L^{r+1}\left(\Sigma_{\lambda}^{w}\right)} \leq \frac{1}{4}\left(\left\|u-u_{\lambda}\right\|_{L^{p+1}\left(\Sigma_{\lambda}^{u}\right)}+\left\|v-v_{\lambda}\right\|_{L^{q+1}\left(\Sigma_{\lambda}^{\nu}\right)}\right) .
$$

Therefore

$$
\begin{aligned}
& \left\|u-u_{\lambda}\right\|_{L^{p+1}\left(\Sigma_{\lambda}^{u}\right)}+\left\|v-v_{\lambda}\right\|_{L^{q+1}\left(\Sigma_{\lambda}^{\nu}\right)}+\left\|w-w_{\lambda}\right\|_{L^{r+1}\left(\Sigma_{\lambda}^{w}\right)} \\
& \quad \leq \frac{1}{2}\left(\left\|u-u_{\lambda}\right\|_{L^{p+1}\left(\Sigma_{\lambda}^{u}\right)}+\left\|v-v_{\lambda}\right\|_{L^{q+1}\left(\Sigma_{\lambda}^{\nu}\right)}+\left\|w-w_{\lambda}\right\|_{L^{r+1}\left(\Sigma_{\lambda}^{w}\right)}\right) .
\end{aligned}
$$

This implies

$$
\left\|u-u_{\lambda}\right\|_{L^{p+1}\left(\Sigma_{\lambda}^{u}\right)}=\left\|v-v_{\lambda}\right\|_{L^{q+1}\left(\Sigma_{\lambda}^{\nu}\right)}=\left\|w-w_{\lambda}\right\|_{L^{r+1}\left(\Sigma_{\lambda}^{w}\right)}=0 .
$$

That is, $\Sigma_{\lambda}^{u}, \Sigma_{\lambda}^{v}$, and $\Sigma_{\lambda}^{w}$ are zero-measure sets. Define

$$
\lambda_{0}:=\sup \left\{\lambda \mid u_{\lambda}-u \geq 0, v_{\lambda}-v \geq 0, w_{\lambda}-w \geq 0, \forall x \in \Sigma_{\lambda}\right\} .
$$

Obviously $\lambda_{0} \leq 0$, by the same method in [16], we can get $\lambda_{0}=0$ and the proof of Theorem 1.1 is complete.

\section{Proof of Theorem 1.2}

In this section, we show that any solution triple of $(1.1)$ in $L^{p+1}\left(\mathbb{R}^{n}\right) \times L^{q+1}\left(\mathbb{R}^{n}\right) \times L^{r+1}\left(\mathbb{R}^{n}\right)$ belongs to $L^{\infty}\left(\mathbb{R}^{n}\right) \times L^{\infty}\left(\mathbb{R}^{n}\right) \times L^{\infty}\left(\mathbb{R}^{n}\right)$. To this purpose, we will use the regularity method developed in [7], which we will state as follows. Let $Z$ be a given vector space, $\|\cdot\|_{X}$ and $\|\cdot\|_{Y}$ be two norms on $Z$. Define a new norm $\|\cdot\|_{Z}$ by

$$
\|\cdot\|_{Z}=\left(\|\cdot\|_{X}^{s}+\|\cdot\|_{Y}^{s}\right)^{\frac{1}{s}} .
$$

Suppose that $Z$ is complete with respect to the norm $\|\cdot\|_{Z}$. Let $X$ and $Y$ be the completion under $\|\cdot\|_{X}$ and $\|\cdot\|_{Y}$, respectively. Here one can choose $s$ such that $1 \leq s \leq \infty$. According to what one needs, it is easy to see that $Z=X \cap Y$. The following regularity lifting theorem was obtained in [7]. 
Lemma 3.1 (Regularity lifting) Let $T$ be a contracting map from $X$ into itself and from $Y$ into itself. Assume that $f \in X$ and that there exists a function $g \in Z$ such that $f=T f+g$, then $f$ also belongs to $Z$.

Proof of Theorem 1.2 Let $(u, v, w) \in L^{p+1}\left(\mathbb{R}^{n}\right) \times L^{q+1}\left(\mathbb{R}^{n}\right) \times L^{r+1}\left(\mathbb{R}^{n}\right)$ be a triple of solution to integral systems (1.1). We first show by Lemma 3.1 that $\left(u_{\xi}, v_{\xi}, w_{\xi}\right)$, a cut-off function of $(u, v, w)$ defined below, belongs to $L^{\tilde{p}}\left(\mathbb{R}^{n}\right) \times L^{\tilde{q}}\left(\mathbb{R}^{n}\right) \times L^{\tilde{r}}\left(\mathbb{R}^{n}\right)$ for $\tilde{p}, \tilde{q}, \tilde{r}>1$ satisfying

$$
\frac{1}{\tilde{p}}-\frac{1}{\tilde{q}}=\frac{1}{p+1}-\frac{1}{q+1}, \quad \frac{1}{\tilde{q}}-\frac{1}{\tilde{r}}=\frac{1}{q+1}-\frac{1}{r+1}, \quad \frac{1}{\tilde{r}}-\frac{1}{\tilde{p}}=\frac{1}{r+1}-\frac{1}{p+1},
$$

then we prove that $(u, v, w) \in L^{\infty}\left(\mathbb{R}^{n}\right) \times L^{\infty}\left(\mathbb{R}^{n}\right) \times L^{\infty}\left(\mathbb{R}^{n}\right)$. For any sufficient large positive real number $\xi$, define

$$
\begin{cases}u_{\xi}(x)=u(x), & \text { if }|u(x)| \geq \xi \text { or }|x|>\xi, \\ u_{\xi}(x)=0, & \text { otherwise. }\end{cases}
$$

Similarly, we may define $v_{\xi}$ and $w_{\xi}$. Let

$$
\begin{aligned}
& \left(T_{1}^{\xi} g\right)(x)=\int_{\mathbb{R}^{n}} \frac{G_{\alpha}(x-y) v_{\xi}^{q-1}(y) w_{\xi}^{r}(y) g(y)}{|y|^{\beta}} d y, \\
& \left(T_{2}^{\xi} h\right)(x)=\int_{\mathbb{R}^{n}} \frac{G_{\alpha}(x-y) w_{\xi}^{r-1}(y) u_{\xi}^{p}(y) h(y)}{|y|^{\beta}} d y, \\
& \left(T_{3}^{\xi} f\right)(x)=\int_{\mathbb{R}^{n}} \frac{G_{\alpha}(x-y) u_{\xi}^{p-1}(y) v_{\xi}^{q}(y) f(y)}{|y|^{\beta}} d y
\end{aligned}
$$

and

$$
T_{\xi}(f, g, h)=\left(T_{1}^{\xi} g, T_{2}^{\xi} h, T_{3}^{\xi} f\right)
$$

Denote $\tilde{u}_{\xi}(x)=u(x)-u_{\xi}(x), E_{\xi}^{u}=\left\{x \in \mathbb{R}^{n}:|u(x)| \geq \xi\right.$ or $\left.|x|>\xi\right\} . \tilde{v}_{\xi}, \tilde{w}_{\xi}$, and $E_{\xi}^{v}, E_{\xi}^{w}$ can be defined in the same way.

By (1.1), we have

$$
\begin{aligned}
u(x)= & \int_{\mathbb{R}^{n}} \frac{G_{\alpha}(x-y) w^{r}(y) v^{q}(y)}{|y|^{\beta}} d y \\
= & \int_{\mathbb{R}^{n}} \frac{G_{\alpha}(x-y) w^{r}(y) v_{\xi}^{q}(y)}{|y|^{\beta}} d y+\int_{\mathbb{R}^{n}} \frac{G_{\alpha}(x-y) w^{r}(y) \tilde{v}_{\xi}^{q}(y)}{|y|^{\beta}} d y \\
= & \int_{\mathbb{R}^{n}} \frac{G_{\alpha}(x-y) w_{\xi}^{r}(y) v_{\xi}^{q}(y)}{|y|^{\beta}} d y+\int_{\mathbb{R}^{n}} \frac{G_{\alpha}(x-y) \tilde{w}_{\xi}^{r}(y) v_{\xi}^{q}(y)}{|y|^{\beta}} d y \\
& +\int_{\mathbb{R}^{n}} \frac{G_{\alpha}(x-y) w_{\xi}^{r}(y) \tilde{v}_{\xi}^{q}(y)}{|y|^{\beta}} d y+\int_{\mathbb{R}^{n}} \frac{G_{\alpha}(x-y) \tilde{w}_{\xi}^{r}(y) \tilde{v}_{\xi}^{q}(y)}{|y|^{\beta}} d y .
\end{aligned}
$$

Thus,

$$
u_{\xi}(x)=\int_{\mathbb{R}^{n}} \frac{G_{\alpha}(x-y) w_{\xi}^{r}(y) v_{\xi}^{q}(y)}{|y|^{\beta}} d y+M_{1}(x)
$$


where

$$
\begin{aligned}
M_{1}(x)= & \int_{\mathbb{R}^{n}} \frac{G_{\alpha}(x-y) \tilde{w}_{\xi}^{r}(y) \nu_{\xi}^{q}(y)}{|y|^{\beta}} d y+\int_{\mathbb{R}^{n}} \frac{G_{\alpha}(x-y) w_{\xi}^{r}(y) \tilde{v}_{\xi}^{q}(y)}{|y|^{\beta}} d y \\
& +\int_{\mathbb{R}^{n}} \frac{G_{\alpha}(x-y) \tilde{w}_{\xi}^{r}(y) \tilde{v}_{\xi}^{q}(y)}{|y|^{\beta}} d y-\tilde{u}_{\xi}(x) .
\end{aligned}
$$

Similarly,

$$
\begin{aligned}
& v_{\xi}(x)=\int_{\mathbb{R}^{n}} \frac{G_{\alpha}(x-y) w_{\xi}^{r}(y) u_{\xi}^{p}(y)}{|y|^{\beta}} d y+M_{2}(x), \\
& w_{\xi}(x)=\int_{\mathbb{R}^{n}} \frac{G_{\alpha}(x-y) u_{\xi}^{p}(y) v_{\xi}^{q}(y)}{|y|^{\beta}} d y+M_{3}(x),
\end{aligned}
$$

where

$$
\begin{aligned}
M_{2}(x)= & \int_{\mathbb{R}^{n}} \frac{G_{\alpha}(x-y) \tilde{u}_{\xi}^{p}(y) w_{\xi}^{r}(y)}{|y|^{\beta}} d y+\int_{\mathbb{R}^{n}} \frac{G_{\alpha}(x-y) u_{\xi}^{p}(y) \tilde{w}_{\xi}^{r}(y)}{|y|^{\beta}} d y \\
& +\int_{\mathbb{R}^{n}} \frac{G_{\alpha}(x-y) \tilde{w}_{\xi}^{r}(y) \tilde{u}_{\xi}^{p}(y)}{|y|^{\beta}} d y-\tilde{v}_{\xi}(x)
\end{aligned}
$$

and

$$
\begin{aligned}
M_{3}(x)= & \int_{\mathbb{R}^{n}} \frac{G_{\alpha}(x-y) \tilde{v}_{\xi}^{q}(y) u_{\xi}^{p}(y)}{|y|^{\beta}} d y+\int_{\mathbb{R}^{n}} \frac{G_{\alpha}(x-y) v_{\xi}^{q}(y) \tilde{u}_{\xi}^{p}(y)}{|y|^{\beta}} d y \\
& +\int_{\mathbb{R}^{n}} \frac{G_{\alpha}(x-y) \tilde{u}_{\xi}^{p}(y) \tilde{v}_{\xi}^{q}(y)}{|y|^{\beta}} d y-\tilde{w}_{\xi}(x) .
\end{aligned}
$$

Therefore

$$
\left(u_{\xi}, v_{\xi}, w_{\xi}\right)=T_{\xi}\left(u_{\xi}, v_{\xi}, w_{\xi}\right)+\left(M_{1}(x), M_{2}(x), M_{3}(x)\right) .
$$

Now, we show that $T_{\xi}(f, g, h)$ is a contraction map from $L^{\tilde{p}}\left(\mathbb{R}^{n}\right) \times L^{\tilde{q}}\left(\mathbb{R}^{n}\right) \times L^{\tilde{r}}\left(\mathbb{R}^{n}\right)$ into $L^{\tilde{p}}\left(\mathbb{R}^{n}\right) \times L^{\tilde{q}}\left(\mathbb{R}^{n}\right) \times L^{\tilde{r}}\left(\mathbb{R}^{n}\right)$ for $\tilde{p}, \tilde{q}, \tilde{r}$ satisfying

$$
\frac{1}{\tilde{p}}-\frac{1}{\tilde{q}}=\frac{1}{p+1}-\frac{1}{q+1}, \quad \frac{1}{\tilde{q}}-\frac{1}{\tilde{r}}=\frac{1}{q+1}-\frac{1}{r+1}, \quad \frac{1}{\tilde{r}}-\frac{1}{\tilde{p}}=\frac{1}{r+1}-\frac{1}{p+1} .
$$

We may verify that $\tilde{q}>\frac{n \tilde{p}}{n+(\alpha-\beta) \tilde{p}}, \tilde{p}>\frac{n \tilde{r}}{n+(\alpha-\beta) \tilde{r}}, \tilde{r}>\frac{n \tilde{q}}{n+(\alpha-\beta) \tilde{q}}$ according to (1.4) and (3.6). Choosing $d_{1}$ such that

$$
\frac{1}{d_{1}}=\frac{q-1}{q+1}+\frac{r}{r+1}+\frac{1}{\tilde{q}}
$$

we may infer by (1.2) that

$$
\tilde{q}>d_{1}>\frac{n \tilde{p}}{n+(\alpha-\beta) \tilde{p}} .
$$


By Hardy-Littlewood-Sobolev's inequality and Hölder's inequality, we find

$$
\left\|T_{1}^{\xi} g\right\|_{\tilde{p}} \leq C\left\|v_{\xi}^{q-1} w_{\xi}^{r} g\right\|_{d_{1}} \leq C\left\|v_{\xi}\right\|_{q+1}^{q-1}\left\|w_{\xi}\right\|_{q+1}^{r}\|g\|_{\tilde{q}}
$$

In the same way, choosing $\frac{1}{d_{2}}=\frac{r-1}{r+1}+\frac{p}{p+1}+\frac{1}{\tilde{r}}$ and $\frac{1}{d_{3}}=\frac{p-1}{p+1}+\frac{q}{q+1}+\frac{1}{\tilde{p}}$, we obtain

$$
\left\|T_{2}^{\xi} h\right\|_{\tilde{q}} \leq C\left\|w_{\xi}^{r-1} u_{\xi}^{p} h\right\|_{d_{2}} \leq C\left\|w_{\xi}\right\|_{r+1}^{r-1}\left\|u_{\xi}\right\|_{p+1}^{p}\|h\|_{\tilde{r}}
$$

and

$$
\left\|T_{3}^{\xi} f\right\|_{\tilde{r}} \leq C\left\|u_{\xi}^{p-1} v_{\xi}^{q} f\right\|_{d_{3}} \leq C\left\|u_{\xi}\right\|_{p+1}^{p-1}\left\|v_{\xi}\right\|_{q+1}^{q}\|f\|_{\tilde{p}}
$$

Since $(u, v, w) \in L^{p+1}\left(\mathbb{R}^{n}\right) \times L^{q+1}\left(\mathbb{R}^{n}\right) \times L^{r+1}\left(\mathbb{R}^{n}\right)$, one can choose $\xi$ sufficiently large so that

$$
C\left\|v_{\xi}\right\|_{q+1}^{q-1}\left\|w_{\xi}\right\|_{q+1}^{r} \leq \frac{1}{2}, \quad C\left\|u_{\xi}\right\|_{p+1}^{p}\left\|w_{\xi}\right\|_{r+1}^{r} \leq \frac{1}{2}, \quad C\left\|u_{\xi}\right\|_{p+1}^{p-1}\left\|v_{\xi}\right\|_{q+1}^{q} \leq \frac{1}{2}
$$

Therefore

$$
\left\|T_{1}^{\xi} g\right\|_{\tilde{p}} \leq \frac{1}{2}\|g\|_{\tilde{q}}, \quad\left\|T_{2}^{\xi} h\right\|_{\tilde{q}} \leq \frac{1}{2}\|h\|_{\tilde{r}}, \quad\left\|T_{3}^{\xi} f\right\|_{\tilde{r}} \leq \frac{1}{2}\|f\|_{\tilde{p}}
$$

and

$$
\begin{aligned}
\left\|T_{\xi}(f, g, h)\right\|_{\tilde{p} \times \tilde{q} \times \tilde{r}} & =\left\|\left(T_{1}^{\xi} g, T_{2}^{\xi} h, T_{3}^{\xi} f\right)\right\|_{\tilde{p} \times \tilde{q} \times \tilde{r}} \\
& =\left\|T_{1}^{\xi} g\right\|_{\tilde{p}}+\left\|T_{2}^{\xi} h\right\|_{\tilde{q}}+\left\|T_{3}^{\xi} f\right\|_{\tilde{r}} \\
& \leq \frac{1}{2}\|g\|_{\tilde{q}}+\frac{1}{2}\|h\|_{\tilde{r}}+\frac{1}{2}\|f\|_{\tilde{p}} \\
& =\frac{1}{2}\|(f, g, h)\|_{\tilde{p} \times \tilde{q} \times \tilde{r}}
\end{aligned}
$$

In other words, $T_{\xi}(f, g, h)$ is a contraction map from $L^{\tilde{p}}\left(\mathbb{R}^{n}\right) \times L^{\tilde{q}}\left(\mathbb{R}^{n}\right) \times L^{\tilde{r}}\left(\mathbb{R}^{n}\right)$ into itself for

$$
\tilde{p}, \tilde{q}, \hat{r}>1, \quad \frac{1}{\tilde{p}}-\frac{1}{\tilde{q}}=\frac{1}{p+1}-\frac{1}{q+1}, \quad \frac{1}{\tilde{p}}-\frac{1}{\tilde{r}}=\frac{1}{p+1}-\frac{1}{r+1}, \quad \frac{1}{\tilde{q}}-\frac{1}{\tilde{r}}=\frac{1}{q+1}-\frac{1}{r+1} .
$$

In particular, for $\tilde{p}=p+1, \tilde{q}=q+1, \tilde{r}=r+1$, we see that $T_{\xi}(f, g, h)$ is also a contraction map from $L^{p+1}\left(\mathbb{R}^{n}\right) \times L^{q+1}\left(\mathbb{R}^{n}\right) \times L^{r+1}\left(\mathbb{R}^{n}\right)$ into itself.

We may assume, without loss of generality, that $q<p<r$. Then $\frac{1}{\tilde{q}}-\frac{1}{\tilde{p}}=\frac{1}{q+1}-\frac{1}{p+1}>0$, and $\tilde{p}>\tilde{q}$. Similarly, $\tilde{r}>\tilde{p}$. Choosing $\tilde{q}$ large such that $\tilde{q}>\frac{n}{\alpha-\beta}$, we also have $\tilde{r}>\tilde{p}>\frac{n}{\alpha-\beta}$. This is possible because we may require $\frac{\alpha-\beta}{n}>\frac{1}{\tilde{q}}>\frac{1}{q+1}-\frac{1}{p+1}$. By Lemma 3.1, we conclude that

$$
\left(u_{\xi}, v_{\xi}, w_{\xi}\right) \in\left(L^{\tilde{p}}\left(\mathbb{R}^{n}\right) \times L^{\tilde{q}}\left(\mathbb{R}^{n}\right) \times L^{\tilde{r}}\left(\mathbb{R}^{n}\right)\right) \cap\left(L^{p+1}\left(\mathbb{R}^{n}\right) \times L^{q+1}\left(\mathbb{R}^{n}\right) \times L^{r+1}\left(\mathbb{R}^{n}\right)\right) .
$$


Let

$$
\begin{aligned}
I_{1}(x)= & \int_{\mathbb{R}^{n}} \frac{G_{\alpha}(x-y) \tilde{w}_{\xi}^{r}(y) v_{\xi}^{q}(y)}{|y|^{\beta}} d y+\int_{\mathbb{R}^{n}} \frac{G_{\alpha}(x-y) w_{\xi}^{r}(y) \tilde{v}_{\xi}^{q}(y)}{|y|^{\beta}} d y \\
& +\int_{\mathbb{R}^{n}} \frac{G_{\alpha}(x-y) \tilde{w}_{\xi}^{r}(y) \tilde{v}_{\xi}^{q}(y)}{|y|^{\beta}} d y \\
:= & J_{1}(x)+J_{2}(x)+J_{3}(x), \\
I_{2}(x)= & \int_{\mathbb{R}^{n}} \frac{G_{\alpha}(x-y) \tilde{u}_{\xi}^{p}(y) w_{\xi}^{r}(y)}{|y|^{\beta}} d y+\int_{\mathbb{R}^{n}} \frac{G_{\alpha}(x-y) u_{\xi}^{p}(y) \tilde{w}_{\xi}^{r}(y)}{|y|^{\beta}} d y \\
& +\int_{\mathbb{R}^{n}} \frac{G_{\alpha}(x-y) \tilde{w}_{\xi}^{r}(y) \tilde{u}_{\xi}^{p}(y)}{|y|^{\beta}} d y, \\
I_{3}(x)= & \int_{\mathbb{R}^{n}} \frac{G_{\alpha}(x-y) \tilde{v}_{\xi}^{q}(y) u_{\xi}^{p}(y)}{|y|^{\beta}} d y+\int_{\mathbb{R}^{n}} \frac{G_{\alpha}(x-y) \nu_{\xi}^{q}(y) \tilde{u}_{\xi}^{p}(y)}{|y|^{\beta}} d y \\
& +\int_{\mathbb{R}^{n}} \frac{G_{\alpha}(x-y) \tilde{u}_{\xi}^{p}(y) \tilde{v}_{\xi}^{q}(y)}{|y|^{\beta}} d y .
\end{aligned}
$$

Next, we claim that $I_{1}, I_{2}, I_{3} \in L^{s}\left(\mathbb{R}^{n}\right)$. We estimate $I_{1}$ only, the estimations for $I_{2}$ and $I_{3}$ can be done in the same way. From (3.7), we need to show $J_{1}, J_{2}, J_{3} \in L^{s}\left(\mathbb{R}^{n}\right)$ to get $I_{1} \in L^{s}\left(\mathbb{R}^{n}\right)$ for any $s>1$.

Condition (1.2) implies $\frac{1}{q+1}>\frac{n-\alpha+\beta}{n}$. Thus, for any $s>1$, we have $\frac{n s}{n+(\alpha-\beta) s}<\frac{q+1}{q}$, which means there exists a $t$ such that $\frac{n s}{n+(\alpha-\beta) s}<t<\frac{q+1}{q}$. By using Hardy-Littlewood-Sobolev's inequality and Hölder's inequality, we get

$$
\left\|J_{1}\right\|_{L^{s}\left(\mathbb{R}^{n}\right)} \leq\left\|v_{\xi}^{q} \tilde{w}_{\xi}^{r}\right\|_{L^{t}\left(\mathbb{R}^{n}\right)} \leq C\left(\int_{\{x:|x| \leq \xi\}} v_{\xi}^{q t}(x) d x\right)^{\frac{1}{t}} \leq C\left\|v_{\xi}\right\|_{q+1}^{q}\left|B_{\xi}(0)\right|^{\frac{1}{t}-\frac{q}{q+1}} \leq C .
$$

Hence, $J_{1} \in L^{s}\left(\mathbb{R}^{n}\right)$ for all $s>1$. The fact $J_{2} \in L^{s}\left(\mathbb{R}^{n}\right)$ can be done in the same way. As for $J_{3}$, it is trivial.

By the fact that $I_{1}, I_{2}, I_{3} \in L^{s}\left(\mathbb{R}^{n}\right)$, and $\tilde{u}_{\xi}, \tilde{v}_{\xi}, \tilde{w}_{\xi}$ belong to $L^{s}\left(\mathbb{R}^{n}\right)$, which is obvious by their definitions, we obtain $M_{1}, M_{2}, M_{3} \in L^{s}\left(\mathbb{R}^{n}\right)$.

Now, we claim that $M_{1}, M_{2}, M_{3} \in L^{\infty}\left(\mathbb{R}^{n}\right)$ for any $s>1$.

It is easy to see from the definition that $\tilde{u}_{\xi}, \tilde{v}_{\xi}, \tilde{w}_{\xi} \in L^{\infty}\left(\mathbb{R}^{n}\right)$. So we need only to show $I_{1}, I_{2}, I_{3} \in L^{\infty}\left(\mathbb{R}^{n}\right)$. Since

$$
J_{1}(x)=\int_{\{y:|w| \leq \xi,|y| \leq \xi\}} \frac{G_{\alpha}(x-y) w^{r}(y) v_{\xi}^{q}(y)}{|y|^{\beta}} d y \leq C \int_{\{y:|y| \leq \xi\}} \frac{G_{\alpha}(x-y) v_{\xi}^{q}(y)}{|y|^{\beta}} d y,
$$

if $x \in \mathbb{R}^{n} \backslash B_{2 \xi}(0), y \in B_{\xi}(0)$, then $|x-y|>|x|-|y|>\xi>|y|$ and

$$
\begin{aligned}
J_{1}(x) & \leq C \int_{\{y:|y| \leq \xi\}} \frac{e^{-\frac{|x-y|}{2}} v_{\xi}^{q}(y)}{|y|^{\beta}} d y \leq C \int_{\{y:|y| \leq \xi\}} \frac{v_{\xi}^{q}(y)}{|y|^{\beta}} d y \\
& \leq C\left(\int_{\{y:|y| \leq \xi\}} v_{\xi}^{q+1}(y) d y\right)^{\frac{q}{q+1}}\left(\int_{\{y:|y| \leq \xi\}} \frac{1}{|y|^{(q+1) \beta}} d y\right)^{\frac{1}{q+1}} \leq C .
\end{aligned}
$$


Chen and Wang Journal of Inequalities and Applications 2014, 2014:222

Page 11 of 21

If $x \in B_{2 \xi}(0)$, then

$$
\begin{aligned}
J_{1}(x) & \leq C \int_{\{y:|y| \leq \xi,|x-y| \geq 2\}} \frac{G_{\alpha}(x-y) v_{\xi}^{q}(y)}{|y|^{\beta}} d y+C \int_{\{y:|y| \leq \xi,|x-y| \leq 2\}} \frac{G_{\alpha}(x-y) v_{\xi}^{q}(y)}{|y|^{\beta}} d y \\
& \leq C \int_{\{y:|y| \leq \xi,|x-y| \geq 2\}} \frac{e^{-\frac{|x-y|}{2}} v_{\xi}^{q}(y)}{|y|^{\beta}} d y+C \int_{\{y:|y| \leq \xi,|x-y| \leq 2\}} \frac{v_{\xi}^{q}(y)}{|x-y|^{n-\alpha}|y|^{\beta}} d y \\
& :=C\left(J_{1,1}(x)+J_{1,2}(x)\right) .
\end{aligned}
$$

Now we estimate $J_{1,1}$ and $J_{1,2}$, respectively,

$$
\begin{aligned}
J_{1,1}(x) & =\int_{\{y:|y| \leq \xi,|x-y| \geq 2\}} \frac{e^{-\frac{|x-y|}{2}} v_{\xi}^{q}(y)}{|y|^{\beta}} d y \\
& \leq C\left(\int_{\{y:|y| \leq \xi\}} v_{\xi}^{q+1}(y) d y\right)^{\frac{q}{q+1}}\left(\int_{\{y:|y| \leq \xi\}} \frac{1}{|y|^{\mid q+1) \beta}} d y\right)^{\frac{1}{q+1}} \leq C
\end{aligned}
$$

and

$$
\begin{aligned}
J_{1,2}(x)= & \int_{\{y: y|\leq \xi,| x-y \mid \leq 2\}} \frac{v_{\xi}^{q}(y)}{|x-y|^{n-\alpha}|y|^{\beta}} d y \\
= & \int_{\{y:|y| \leq \xi,|x-y| \leq 2,|x-y| \geq|y|\}} \frac{v_{\xi}^{q}(y)}{|x-y|^{n-\alpha}|y|^{\beta}} d y \\
& +\int_{\{y:|y| \leq \xi,|x-y| \leq 2,|x-y| \leq|y|\}} \frac{v_{\xi}^{q}(y)}{|x-y|^{n-\alpha}|y|^{\beta}} d y \\
\leq & \int_{\{y:|y| \leq \xi\}} \frac{v_{\xi}^{q}(y)}{|y|^{n-\alpha+\beta}} d y+\int_{\{y:|y| \leq \xi,|x-y| \leq 2\}} \frac{v_{\xi}^{q}(y)}{|x-y|^{n-\alpha+\beta}} d y \\
\leq & \left(\int_{\{y:|y| \leq \xi\}} v_{\xi}^{q+1}(y) d y\right)^{\frac{q}{q+1}}\left(\int_{\{y:|y| \leq \xi\}} \frac{1}{|y|^{(n-\alpha+\beta)(q+1)}} d y\right)^{\frac{1}{q+1}} \\
& +\left(\int_{\{y:|y| \leq \xi\}} v_{\xi}^{q+1}(y) d y\right)^{\frac{q}{q+1}}\left(\int_{\{y:|x-y| \leq 2\}} \frac{1}{|x-y|^{(n-\alpha+\beta)(q+1)}} d y\right)^{\frac{1}{q+1}} \leq C,
\end{aligned}
$$

where we use the fact that $\frac{1}{q+1}>\frac{n-\alpha+\beta}{n}$. Therefore, $J_{1} \in L^{\infty}\left(\mathbb{R}^{n}\right)$. Similarly, we have $J_{2} \in$ $L^{\infty}\left(\mathbb{R}^{n}\right)$.

As for $J_{3}(x)$, we have

$$
\begin{aligned}
J_{3}(x) & =\int_{\mathbb{R}^{n}} \frac{G_{\alpha}(x-y) \tilde{w}_{\xi}^{r}(y) \tilde{v}_{\xi}^{q}(y)}{|y|^{\beta}} d y \leq C \int_{\{y:|y| \leq \xi\}} \frac{G_{\alpha}(x-y)}{|y|^{\beta}} d y \\
& \leq C \int_{\{y:|y| \leq \xi,|x-y| \geq 2\}} \frac{e^{-\frac{|x-y|}{2}}}{|y|^{\beta}} d y+C \int_{\{y:|y| \leq \xi,|x-y|<2\}} \frac{1}{|x-y|^{n-\alpha}|y|^{\beta}} d y \\
& :=J_{3,1}(x)+J_{3,2}(x) .
\end{aligned}
$$

Since

$$
J_{3,1}(x) \leq C \int_{\{y:|y| \leq \xi\}} \frac{1}{|y|^{\beta}} d y \leq C
$$


and

$$
\begin{aligned}
J_{3,2}(x) \leq & C \int_{\{y:|y| \leq \xi,|x-y|<2,|x-y| \geq|y|\}} \frac{1}{|x-y|^{n-\alpha}|y|^{\beta}} d y \\
& +C \int_{\{y:|y| \leq \xi,|x-y|<2,|x-y|<|y|\}} \frac{1}{|x-y|^{n-\alpha}|y|^{\beta}} d y \\
\leq & C \int_{\{y:|y| \leq \xi\}} \frac{1}{|y|^{n-\alpha+\beta}} d y+C \int_{\{y:|x-y|<2\}} \frac{1}{|x-y|^{n-\alpha+\beta}} d y \leq C,
\end{aligned}
$$

we infer $J_{3} \in L^{\infty}\left(\mathbb{R}^{n}\right)$.

Thus, we have shown that $I_{1} \in L^{\infty}\left(\mathbb{R}^{n}\right) \cap L^{s}\left(\mathbb{R}^{n}\right)$ for any $s>1$. Similarly, we can deduce that $I_{2}, I_{3} \in L^{\infty}\left(\mathbb{R}^{n}\right) \cap L^{s}\left(\mathbb{R}^{n}\right)$ for any $s>1$. Consequently, $M_{1}, M_{2}, M_{3} \in L^{\infty}\left(\mathbb{R}^{n}\right) \cap L^{s}\left(\mathbb{R}^{n}\right)$ for any $s>1$.

Finally, we show that $u, v, w \in L^{\infty}\left(\mathbb{R}^{n}\right)$. Since $u(x)=u_{\xi}(x)+\tilde{u}_{\xi}(x), v(x)=v_{\xi}(x)+\tilde{v}_{\xi}(x)$, $w(x)=w_{\xi}(x)+\tilde{w}_{\xi}(x)$, and $\tilde{u}_{\xi}, \tilde{v}_{\xi}, \tilde{w}_{\xi} \in L^{\infty}\left(\mathbb{R}^{n}\right)$, we only need to verify $u_{\xi}, v_{\xi}, w_{\xi} \in L^{\infty}\left(\mathbb{R}^{n}\right)$. By (3.2)-(3.5) and $M_{1}, M_{2}, M_{3} \in L^{\infty}\left(\mathbb{R}^{n}\right)$, it is sufficient to verify that $L_{1}, L_{2}, L_{3} \in L^{\infty}\left(\mathbb{R}^{n}\right)$, where

$$
\begin{aligned}
& L_{1}(x)=\int_{\mathbb{R}^{n}} \frac{G_{\alpha}(x-y) w_{\xi}^{r}(y) v_{\xi}^{q}(y)}{|y|^{\beta}} d y, \quad L_{2}(x)=\int_{\mathbb{R}^{n}} \frac{G_{\alpha}(x-y) w_{\xi}^{r}(y) u_{\xi}^{p}(y)}{|y|^{\beta}} d y, \\
& L_{3}(x)=\int_{\mathbb{R}^{n}} \frac{G_{\alpha}(x-y) u_{\xi}^{p}(y) v_{\xi}^{q}(y)}{|y|^{\beta}} d y .
\end{aligned}
$$

In fact,

$$
\begin{aligned}
L_{1}(x) & =\int_{\{y:|y| \leq \xi\}} \frac{G_{\alpha}(x-y) w_{\xi}^{r} v_{\xi}^{q}}{|y|^{\beta}} d y+\int_{\{y:|y| \geq \xi\}} \frac{G_{\alpha}(x-y) w_{\xi}^{r} v_{\xi}^{q}}{|y|^{\beta}} d y \\
& :=N(x)+K(x) .
\end{aligned}
$$

Choosing $\tilde{q}$ large enough such that $\tilde{q}>\frac{q(p+1)(q+1)}{p q-1}$, that is,

$$
\frac{1}{\tilde{q}}<\frac{1}{q+1}-\frac{1}{q(p+1)}
$$

If $x \in \mathbb{R}^{n} \backslash B_{2 \xi}(0), y \in B_{\xi}(0)$, then $|x-y|>|x|-|y|>\xi>|y|$. Thus,

$$
\begin{aligned}
N(x) & \leq C \int_{\{y:|y| \leq \xi\}} \frac{e^{-\frac{|x-y|}{2}} w_{\xi}^{r} v_{\xi}^{q}}{|y|^{\beta}} d y \leq C \int_{\{y:|y| \leq \xi\}} \frac{w_{\xi}^{r} v_{\xi}^{q}}{|y|^{\beta}} d y \\
& \leq C\left\|w_{\xi}\right\|_{r+1}^{r}\left\|v_{\xi}\right\|_{\tilde{q}}^{q}\left(\int_{\{y:|y| \leq \xi\}} \frac{1}{|y|^{\frac{\beta}{1-\frac{r}{r+1}-\frac{q}{\tilde{q}}}}} d y\right)^{\frac{1}{r+1}-\frac{q}{\tilde{q}}} \leq C,
\end{aligned}
$$

where we use the fact that $\tilde{q}>\frac{q(p+1)(q+1)}{p q-1}>\frac{n q(r+1)}{n-(r+1)(n-\alpha+\beta)}>\frac{n q(r+1)}{n-(r+1) \beta}$. 
If $x \in B_{2 \xi}(0)$, then

$$
\begin{aligned}
N(x) & \leq C \int_{\{y:|y| \leq \xi,|x-y| \geq 2\}} \frac{e^{-\frac{|x-y|}{2}} w_{\xi}^{r} v_{\xi}^{q}}{|y|^{\beta}} d y+C \int_{\{y:|y| \leq \xi,|x-y| \leq 2\}} \frac{w_{\xi}^{r} v_{\xi}^{q}}{|x-y|^{n-\alpha}|y|^{\beta}} d y \\
& :=C\left(N_{1}(x)+N_{2}(x)\right) .
\end{aligned}
$$

Now we estimate $N_{1}(x), N_{2}(x)$, respectively. By Hölder's inequality, we have

$$
\begin{aligned}
N_{1}(x) & \leq C \int_{\{y:|y| \leq \xi\}} \frac{w_{\xi}^{r} v_{\xi}^{q}}{|y|^{\beta}} d y \\
& \leq C\left\|w_{\xi}\right\|_{r+1}^{r}\left\|v_{\xi}\right\|_{\tilde{q}}^{q}\left(\int_{\{y:|y| \leq \xi\}} \frac{1}{|y|^{\frac{r}{r+1-\frac{q}{q}}}} d y\right)^{1-\frac{1}{r+1}-\frac{q}{q}} \leq C
\end{aligned}
$$

since $\tilde{q}>\frac{n q(r+1)}{n-(r+1) \beta}$. On the other hand, we deduce

$$
\begin{aligned}
N_{2}(x)= & \int_{\{y:|y| \leq \xi,|x-y| \leq 2,|x-y| \geq|y|\}} \frac{w_{\xi}^{r} v_{\xi}^{q}}{|x-y|^{n-\alpha}|y|^{\beta}} d y \\
& +\int_{\{y:|y| \leq \xi,|x-y| \leq 2,|x-y| \leq|y|\}} \frac{w_{\xi}^{r} v_{\xi}^{q}}{|x-y|^{n-\alpha}|y|^{\beta}} d y \\
\leq & \int_{\{y:|y| \leq \xi\}} \frac{w_{\xi}^{r} v_{\xi}^{q}}{|y|^{n-\alpha+\beta}} d y+\int_{\{y:|y| \leq \xi,|x-y| \leq 2\}} \frac{w_{\xi}^{r} v_{\xi}^{q}}{|x-y|^{n-\alpha+\beta}} d y \\
\leq & \left\|w_{\xi}\right\|_{r+1}^{r}\left\|v_{\xi}\right\|_{\tilde{q}}^{q}\left(\int_{\{y:|y| \leq \xi\}} \frac{1}{|y|^{\frac{n-\alpha+\beta}{1-\frac{r}{r+1}-\frac{q}{q}}}} d y\right)^{\frac{1}{r+1}-\frac{q}{\bar{q}}} \\
& +\left\|w_{\xi}\right\|_{r+1}^{r}\left\|v_{\xi}\right\|_{\tilde{q}}^{q}\left(\int_{\{y:|x-y| \leq 2\}} \frac{1}{|x-y|^{\frac{n-\alpha+\beta}{1-\frac{r}{r+1}-\frac{q}{\bar{q}}}}} d y\right)^{\frac{1}{r+1}-\frac{q}{\bar{q}}} \\
\leq & C
\end{aligned}
$$

by the fact that $\tilde{q}>\frac{n q(r+1)}{n-(r+1)(n-\alpha+\beta)}$.

Inequalities (3.13)-(3.16) imply that $N(x) \in L^{\infty}\left(\mathbb{R}^{n}\right)$. Now we estimate $K(x)$. For any $x \in$ $\mathbb{R}^{n}$,

$$
\begin{aligned}
K(x) & =\int_{\{y:|y| \geq \xi\}} \frac{G_{\alpha}(x-y) w_{\xi}^{r} v_{\xi}^{q}}{|y|^{\beta}} d y \\
& \leq C \int_{\{y:|y| \geq \xi,|x-y| \geq 2\}} \frac{e^{-\frac{|x-y|}{2}} w_{\xi}^{r} v_{\xi}^{q}}{|y|^{\beta}} d y+C \int_{\{y:|y| \geq \xi,|x-y| \leq 2\}} \frac{w_{\xi}^{r} v_{\xi}^{q}}{|x-y|^{n-\alpha}|y|^{\beta}} d y \\
& \leq C \int_{\{y:|y| \geq \xi,|x-y| \geq 2\}} \frac{e^{-\frac{|x-y|}{2}} w_{\xi}^{r} v_{\xi}^{q}}{|y|^{\beta}} d y+C \int_{\{y:|x-y| \leq 2\}} \frac{w_{\xi}^{r} v_{\xi}^{q}}{|x-y|^{n-\alpha+\beta}} d y \\
& :=C\left(K_{1}(x)+K_{2}(x)\right) .
\end{aligned}
$$


By Hölder's inequality,

$$
K_{2}(x) \leq\left\|w_{\xi}\right\|_{r+1}^{r}\left\|v_{\xi}\right\|_{\tilde{q}}^{q}\left(\int_{\{y:|x-y| \leq 2\}} \frac{1}{|x-y|^{\frac{n-\alpha+\beta}{1-\frac{r}{r+1}-\frac{q}{q}}}} d y\right)^{\frac{1}{r+1}-\frac{q}{q}} \leq C .
$$

Now we estimate $K_{1}(x)$. Using the fact that $\tilde{q}>\frac{q(p+1)(q+1)}{p q-1}>\frac{n q(r+1)}{n-(r+1)(n-\alpha+\beta)}$, we can choose a $t_{1}$ such that $1<t_{1}<\frac{n(r+1) \tilde{q}}{(n-\beta(r+1)) \tilde{q}-n q(r+1)}$. Hence, Hölder's inequality implies that

$$
\begin{aligned}
K_{1}(x) \leq & \left\|w_{\xi}\right\|_{r+1}^{r}\left\|v_{\xi}\right\|_{\tilde{q}}^{q}\left(\int_{\{y:|x-y| \geq 2\}} e^{-\frac{|x-y| t_{1}}{2}} d y\right)^{\frac{1}{t_{1}}} \\
& \times\left(\int_{\{y:|y| \geq \xi\}} \frac{1}{|y|^{\frac{r}{r+1}-\frac{q}{q}-\frac{1}{t_{1}}}} d y\right)^{\frac{1}{r+1}-\frac{q}{q}-\frac{1}{t_{1}}} \leq C .
\end{aligned}
$$

Consequently, both $N$ and $K$ belong to $L^{\infty}\left(\mathbb{R}^{n}\right)$, so we have $L_{1}$.

By (1.2), (3.6), (3.12), and the assumption that $q<p<r$, we see that $\tilde{p}, \tilde{r}$ satisfies

$$
\begin{aligned}
\frac{1}{\tilde{p}} & <\frac{1}{p+1}-\frac{1}{(p+1) q}<\frac{1}{p+1}-\frac{1}{(r+1) p} \\
& =\frac{1}{p}\left(1-\frac{1}{p+1}-\frac{1}{r+1}\right)<\frac{1}{p}\left(\frac{1}{q+1}-\frac{n-\alpha+\beta}{n}\right), \\
\frac{1}{\tilde{r}} & <\frac{1}{r+1}-\frac{1}{(p+1) q}<\frac{1}{r+1}-\frac{1}{(q+1) r} \\
& =\frac{1}{r}\left(1-\frac{1}{r+1}-\frac{1}{q+1}\right)<\frac{1}{r}\left(\frac{1}{p+1}-\frac{n-\alpha+\beta}{n}\right) .
\end{aligned}
$$

Inequalities (3.19) and (3.20) allow us to show in the same way that $L_{2}, L_{3} \in L^{\infty}\left(\mathbb{R}^{n}\right)$. Consequently, $(u, v, w) \in L^{\infty}\left(\mathbb{R}^{n}\right) \times L^{\infty}\left(\mathbb{R}^{n}\right) \times L^{\infty}\left(\mathbb{R}^{n}\right)$. Theorem 1.2 is proved.

\section{Hölder continuity}

We will show in this section that solution triples of (1.1) are Hölder continuous by regularity lifting Theorem II in [7]. We first recall the theorem.

Let $V$ be a Hausdorff topological vector space. Suppose there are two extended norms defined on $V,\|\cdot\|_{X},\|\cdot\|_{Y}: V \rightarrow[0, \infty]$. Let

$$
X:=\left\{v \in V:\|v\|_{X}<\infty\right\}, \quad Y:=\left\{v \in V:\|v\|_{Y}<\infty\right\} .
$$

We also assume that $X$ is complete and that the topology in $V$ is weaker than the topology of $X$ and the weak topology of $Y$, which means that the convergence in $X$ or weak convergence in $Y$ will imply convergence in $V$. The pair of spaces $(X, Y)$ described as above is called an $X Y$-pair, if whenever the sequence $\left\{u_{n}\right\} \subset X$ with $u_{n} \rightarrow u$ in $X$ and $\left\|u_{n}\right\|_{Y} \leq C$ will imply $u \in Y$.

It is known from Remark 3.3.5 in [7] that if $X=L^{p}(U)$ for $1 \leq p \leq \infty, Y=C^{0, \gamma}(U)$ for $0<\gamma \leq 1$, and $V$ is the space of distributions, where $U$ can be any subset of $\mathbb{R}^{n}$ or $\mathbb{R}^{n}$ itself, then $(X, Y)$ is an $X Y$-pair. 
Lemma 4.1 (Regularity lifting II [7]) Let X, Y be Banach spaces contained in some larger topological space $V$ satisfying properties described above, and that $\mathfrak{X}$ and $\mathfrak{Y}$ be closed subsets of $X$ and $Y$, respectively. Suppose that $(X, Y)$ is an $X Y$-pair, $T: \mathfrak{X} \rightarrow X$ is a contraction:

$$
\|T f-T g\|_{X} \leq \eta\|f-g\|_{\mathfrak{X}}, \quad \forall f, g \in \mathfrak{X} \text { and for some } 0<\eta<1 ;
$$

and $T: \mathfrak{Y} \rightarrow Y$ is shrinking:

$$
\|T g\|_{Y} \leq \theta\|g\|_{\mathfrak{Y}}, \quad \forall g \in \mathfrak{Y} \text { and for some } 0<\theta<1 .
$$

Define

$$
S f=T f+F \quad \text { for some } F \in \mathfrak{X} \cap \mathfrak{Y} \text {. }
$$

Moreover, assume that

$$
S: \mathfrak{X} \cap \mathfrak{Y} \rightarrow \mathfrak{X} \cap \mathfrak{Y} .
$$

Then there exists a unique solution $u$ of equation

$$
u=T f+F \quad \text { in } \mathfrak{X},
$$

and, more importantly,

$$
u \in Y .
$$

Proof of Theorem 1.3 Since

$$
-\int_{0}^{\infty} G_{\alpha}^{\prime}(t) \int_{B_{t}(x)} \frac{w^{r}(y) v^{q}(y)}{|y|^{\beta}} d y d t=\int_{\mathbb{R}^{n}} \frac{G_{\alpha}(x-y) w^{r}(y) v^{q}(y)}{|y|^{\beta}} d y,
$$

the solution triples $(u, v, w)$ of $(1.1)$ are solutions of the following system:

$$
\left\{\begin{array}{l}
u(x)=-\int_{0}^{\infty} G_{\alpha}^{\prime}(t) \int_{B_{t}(x)} \frac{w^{r}(y) v^{q}(y)}{|y| \beta} d y d t, \\
v(x)=-\int_{0}^{\infty} G_{\alpha}^{\prime}(t) \int_{B_{t}(x)} \frac{u^{p}(y) w^{r}(y)}{|y|^{\beta}} d y d t, \\
w(x)=-\int_{0}^{\infty} G_{\alpha}^{\prime}(t) \int_{B_{t}(x)} \frac{u^{p}(y) v^{q}(y)}{|y|^{\beta}} d y d t .
\end{array}\right.
$$

Hence, it is sufficient to prove that the solutions of (4.1) are Hölder continuous.

For any $\Omega \subset \subset \mathbb{R}^{n} \backslash\{0\}$, denote $d=\operatorname{dist}(0, \Omega)>0$. Let $X=L^{\infty}(\Omega)$ and $Y=C^{0, \gamma}(\Omega)$, where $\gamma=1-\frac{\beta}{n}$. By Theorem 1.2, $u, v, w \in L^{\infty}\left(\mathbb{R}^{n}\right)$, so we may define

$$
\begin{aligned}
& \mathfrak{X}=\left\{g \in X \mid\|g\|_{\infty} \leq 2\|u\|_{\infty}+2\|v\|_{L^{\infty}}+2\|w\|_{\infty}\right\}, \\
& \mathfrak{Y}=\left\{g \in Y \mid\|g\|_{\infty} \leq 2\|u\|_{\infty}+2\|v\|_{L^{\infty}}+2\|w\|_{\infty}\right\} .
\end{aligned}
$$


For every $0<\varepsilon<\frac{d}{2}$, we define

$$
\begin{aligned}
& T_{1}^{\varepsilon}(g, h)(x)=-\int_{0}^{\varepsilon} G_{\alpha}^{\prime}(t) \int_{B_{t}(x)} \frac{h^{r}(y) g^{q}(y)}{|y|^{\beta}} d y d t, \\
& T_{2}^{\varepsilon}(h, f)(x)=-\int_{0}^{\varepsilon} G_{\alpha}^{\prime}(t) \int_{B_{t}(x)} \frac{f^{p}(y) h^{r}(y)}{|y|^{\beta}} d y d t, \\
& T_{3}^{\varepsilon}(f, g)(x)=-\int_{0}^{\varepsilon} G_{\alpha}^{\prime}(t) \int_{B_{t}(x)} \frac{g^{q}(y) f^{p}(y)}{|y|^{\beta}} d y d t
\end{aligned}
$$

and

$$
T_{\varepsilon}(f, g, h)=\left(T_{1}^{\varepsilon}(g, h), T_{2}^{\varepsilon}(h, f), T_{3}^{\varepsilon}(f, g)\right) .
$$

Furthermore, we define

$$
\begin{aligned}
& U(x)=-\int_{\varepsilon}^{\infty} G_{\alpha}^{\prime}(t) \int_{B_{t}(x)} \frac{w^{r}(y) v^{q}(y)}{|y|^{\beta}} d y d t, \\
& V(x)=-\int_{\varepsilon}^{\infty} G_{\alpha}^{\prime}(t) \int_{B_{t}(x)} \frac{u^{p}(y) w^{r}(y)}{|y|^{\beta}} d y d t, \\
& W(x)=-\int_{\varepsilon}^{\infty} G_{\alpha}^{\prime}(t) \int_{B_{t}(x)} \frac{v^{q}(y) u^{p}(y)}{|y|^{\beta}} d y d t .
\end{aligned}
$$

Obviously, a solution $(u, v, w)$ of $(4.1)$ is a solution of the equation

$$
(u, v, w)=T_{\varepsilon}(u, v, w)+G
$$

where $G=(U, V, W)$. Write

$$
S_{\varepsilon}(u, v, w)=T_{\varepsilon}(u, v, w)+G
$$

We will show for $\varepsilon>0$ small that

$$
T_{\varepsilon}: \mathfrak{X} \times \mathfrak{X} \times \mathfrak{X} \rightarrow X \times X \times X
$$

is a contracting operator and

$$
T_{\varepsilon}: \mathfrak{Y} \times \mathfrak{Y} \times \mathfrak{Y} \rightarrow Y \times Y \times Y
$$

is a shrinking operator. Furthermore,

$$
G \in(\mathfrak{X} \times \mathfrak{X} \times \mathfrak{X}) \cap(\mathfrak{Y} \times \mathfrak{Y} \times \mathfrak{Y}),
$$

and

$$
S_{\varepsilon}:(\mathfrak{X} \times \mathfrak{X} \times \mathfrak{X}) \cap(\mathfrak{Y} \times \mathfrak{Y} \times \mathfrak{Y}) \rightarrow(\mathfrak{X} \times \mathfrak{X} \times \mathfrak{X}) \cap(\mathfrak{Y} \times \mathfrak{Y} \times \mathfrak{Y}) .
$$

This then will yield $(u, v, w) \in Y \times Y \times Y$ by Lemma 4.1. 
We first show that $T_{\varepsilon}$ is a contracting operator from $\mathfrak{X} \times \mathfrak{X} \times \mathfrak{X}$ to $X \times X \times X$. We have $\left(g_{1}, h_{1}\right),\left(g_{2}, h_{2}\right) \in \mathfrak{X} \times \mathfrak{X}$. By the mean value theorem, we have

$$
\begin{aligned}
& \left|T_{1}^{\varepsilon}\left(g_{1}, h_{1}\right)(x)-T_{1}^{\varepsilon}\left(g_{2}, h_{2}\right)(x)\right| \\
& \leq\left|\int_{0}^{\varepsilon} G_{\alpha}^{\prime}(t) \int_{B_{t}(x)} \frac{h_{1}^{r}(y) g_{1}^{q}(y)-h_{2}^{r}(y) g_{2}^{q}(y)}{|y|^{\beta}} d y d t\right| \\
& \leq \int_{0}^{\varepsilon} G_{\alpha}^{\prime}(t) \int_{B_{t}(x)}\left|\frac{\left(h_{1}^{r}(y)-h_{2}^{r}(y)\right) g_{1}^{q}(y)+h_{2}^{r}(y)\left(g_{1}^{q}(y)-g_{2}^{q}(y)\right)}{|y|^{\beta}}\right| d y d t \\
& \leq\left\|g_{1}\right\|_{\infty}^{q} \max \left\{\left\|h_{1}\right\|_{\infty}^{r-1},\left\|h_{2}\right\|_{\infty}^{r-1}\right\}\left\|h_{1}-h_{2}\right\|_{\infty} \int_{0}^{\varepsilon}\left|G_{\alpha}^{\prime}(t)\right| \int_{0}^{t} r^{n-\beta-1} d r d t \\
& \quad+\left\|h_{2}\right\|_{\infty}^{r} \max \left\{\left\|g_{1}\right\|_{\infty}^{q-1},\left\|g_{2}\right\|_{\infty}^{q-1}\right\}\left\|g_{1}-g_{2}\right\|_{\infty} \int_{0}^{\varepsilon}\left|G_{\alpha}^{\prime}(t)\right| \int_{0}^{t} r^{n-\beta-1} d r d t \\
& \leq C\left(\left\|h_{1}-h_{2}\right\|_{\infty}+\left\|g_{1}-g_{2}\right\|_{\infty}\right) \varepsilon^{\alpha-\beta},
\end{aligned}
$$

where we use the fact that

$$
\int_{0}^{\varepsilon} s^{n-\beta}\left|G_{\alpha}^{\prime}(s)\right| d s=O\left(\varepsilon^{\alpha-\beta}\right) .
$$

Choosing $\varepsilon>0$ small so that $C \varepsilon^{\alpha-\beta}<\frac{1}{4}$, we see that $T_{1}^{\varepsilon}$ is a contracting operator from $\mathfrak{X} \times \mathfrak{X}$ to $X \times X$. Similarly, $T_{2}^{\varepsilon}$ and $T_{3}^{\varepsilon}$ are also contracting operators from $\mathfrak{X} \times \mathfrak{X}$ to $X \times X$. Therefore, $T_{\varepsilon}$ is a contracting operator from $\mathfrak{X} \times \mathfrak{X} \times \mathfrak{X}$ to $X \times X \times X$.

Next, we verify that $T_{\varepsilon}$ is a shrinking operator from $\mathfrak{Y} \times \mathfrak{Y} \times \mathfrak{Y}$ to $Y \times Y \times Y$. We only show it for $T_{1}^{\varepsilon}$; it can be done in the same way for $T_{2}^{\varepsilon}$ and $T_{3}^{\varepsilon}$. For any $x, z \in \Omega$ and $(g, h) \in \mathfrak{Y} \times \mathfrak{Y}$ we have

$$
\begin{aligned}
\left|T_{1}^{\varepsilon}(g, h)(x)-T_{1}^{\varepsilon}(g, h)(z)\right| & \\
\leq & \int_{0}^{\varepsilon}\left|G_{\alpha}^{\prime}(t)\right|\left|\int_{B_{t}(x)} \frac{g^{q}(y) h^{r}(y)}{|y|^{\beta}} d y-\int_{B_{t}(z)} \frac{g^{q}(y) h^{r}(y)}{|y|^{\beta}} d y\right| d t \\
= & \int_{0}^{\varepsilon}\left|G_{\alpha}^{\prime}(t)\right|\left|\int_{B_{t}(x)} \frac{h^{r}(y) g^{q}(y)}{|y|^{\beta}} d y-\int_{B_{t}(x)} \frac{h^{r}(y+z-x) g^{q}(y+z-x)}{|y+z-x|^{\beta}} d y\right| d t \\
\leq & \int_{0}^{\varepsilon}\left|G_{\alpha}^{\prime}(t)\right|\left|\int_{B_{t}(x)} \frac{h^{r}(y) g^{q}(y)}{|y|^{\beta}} d y-\int_{B_{t}(x)} \frac{h^{r}(y) g^{q}(y)}{|y+z-x|^{\beta}} d y\right| d t \\
& +\int_{0}^{\varepsilon}\left|G_{\alpha}^{\prime}(t)\right|\left|\int_{B_{t}(x)} \frac{h^{r}(y) g^{q}(y)-h^{r}(y+z-x) g^{q}(y+z-x)}{|y+z-x|^{\beta}} d y\right| d t \\
= & : A(x)+B(x) .
\end{aligned}
$$

For $y \in B_{t}(x), 0<t<\varepsilon$, we have $|y| \geq|x|-t \geq d-\frac{d}{2}=\frac{d}{2}$ and $|y+z-x| \geq|z|-|y-x| \geq$ $d-t \geq \frac{d}{2}$. So both $\frac{1}{|y|^{\beta}}$ and $\frac{1}{|y+z-x|^{\beta}}$ are regular in $B_{t}(x)$ for $0<t<\varepsilon$. In particular, there exists $C>0$ such that $\left|\frac{1}{|y|^{\beta}}-\frac{1}{|y+z-x|^{\beta}}\right| \leq C|x-z|$. Hence,

$$
\begin{aligned}
A(x) & =\int_{0}^{\varepsilon}\left|G_{\alpha}^{\prime}(t)\right|\left|\int_{B_{t}(x)} h^{r}(y) g^{q}(y)\left(\frac{1}{|y|^{\beta}}-\frac{1}{|y+z-x|^{\beta}}\right) d y\right| d t \\
& \leq C\|g\|_{\infty}^{q}\|h\|_{\infty}^{r}|x-z| \int_{0}^{\varepsilon}\left|G_{\alpha}^{\prime}(t)\right| t^{n} d t=C\|g\|_{\infty}^{q}\|h\|_{\infty}^{r}|x-z| \varepsilon^{\alpha} .
\end{aligned}
$$


If $|x-z| \leq 1,|x-z| \leq|x-z|^{\gamma}$; if $|x-z|>1,|x-z| \leq(\operatorname{diam} \Omega)^{1-\gamma}|x-z|^{\gamma}$. Therefore,

$$
A(x) \leq C\|g\|_{\infty}^{q}\|h\|_{\infty}^{r-1}\|h\|_{C^{0, \gamma}}|x-z|^{\gamma} \varepsilon^{\alpha}
$$

On the other hand, by the mean value theorem,

$$
\begin{aligned}
& B(x)=\int_{0}^{\varepsilon}\left|G_{\alpha}^{\prime}(t)\right|\left|\int_{B_{t}(x)} \frac{h^{r}(y) g^{q}(y)-h^{r}(y+z-x) g^{q}(y+z-x)}{|y+z-x|^{\beta}} d y\right| d t \\
& \leq \int_{0}^{\varepsilon}\left|G_{\alpha}^{\prime}(t)\right|\left|\int_{B_{t}(x)} \frac{\left(g^{q}(y)-g^{q}(y+z-x)\right) h^{r}(y)}{|y+z-x|^{\beta}} d y\right| d t \\
& +\int_{0}^{\varepsilon}\left|G_{\alpha}^{\prime}(t)\right|\left|\int_{B_{t}(x)} \frac{\left(h^{r}(y)-h^{r}(y+z-x)\right) g^{q}(y+z-x)}{|y+z-x|^{\beta}} d y\right| d t \\
& \leq C\left(\frac{2}{d}\right)^{\beta}\left(\|g\|_{\infty}^{q-1}\|h\|_{\infty}^{r}\|g\|_{C^{0, \gamma}}|x-z|^{\gamma}\right) \int_{0}^{\varepsilon}\left|G_{\alpha}^{\prime}(t)\right| t^{n} d t \\
& +C\left(\frac{2}{d}\right)^{\beta}\left(\|g\|_{\infty}^{q}\|h\|_{\infty}^{r-1}\|h\|_{C^{0, \gamma}}|x-z|^{\gamma}\right) \int_{0}^{\varepsilon}\left|G_{\alpha}^{\prime}(t)\right| t^{n} d t \\
& \leq C\left(\|g\|_{\infty}^{q-1}\|h\|_{\infty}^{r}\|g\|_{C^{0, \gamma}}|x-z|^{\gamma}+\|g\|_{\infty}^{q}\|h\|_{\infty}^{r-1}\|h\|_{C^{0, \gamma}}|x-z|^{\gamma}\right) \varepsilon^{\alpha} .
\end{aligned}
$$

So

$$
\left|T_{1}^{\varepsilon}(g, h)(x)-T_{1}^{\varepsilon}(g, h)(z)\right| \leq C\left(\|g\|_{C^{0, \gamma}}|x-z|^{\gamma}+\|h\|_{C^{0, \gamma}}|x-z|^{\gamma}\right) \varepsilon^{\alpha}
$$

Choosing $\varepsilon$ sufficiently small, we obtain

$$
\sup _{x \neq z} \frac{\left|T_{1}^{\varepsilon}(g, h)(x)-T_{1}^{\varepsilon}(g, h)(z)\right|}{|x-z|^{\gamma}} \leq \frac{1}{4}\left(\|g\|_{C^{0, \gamma}}+\|h\|_{C^{0, \gamma}}\right) .
$$

Similarly, we have

$$
\sup _{x \neq z} \frac{\left|T_{2}^{\varepsilon}(h, f)(x)-T_{2}^{\varepsilon}(h, f)(z)\right|}{|x-z|^{\gamma}} \leq \frac{1}{4}\left(\|h\|_{C^{0, \gamma}}+\|f\|_{C^{0, \gamma}}\right)
$$

and

$$
\sup _{x \neq z} \frac{\left|T_{3}^{\varepsilon}(f, g)(x)-T_{3}^{\varepsilon}(f, g)(z)\right|}{|x-z|^{\gamma}} \leq \frac{1}{4}\left(\|f\|_{C^{0, \gamma}}+\|g\|_{C^{0, \gamma}}\right) .
$$

Consequently,

$$
\sup _{x \neq z} \frac{\left|T_{\varepsilon}(f, g, h)(x)-T_{\varepsilon}(f, g, h)(z)\right|}{|x-z|^{\gamma}} \leq \frac{1}{2}\|(f, g, h)\|_{C^{0, \gamma}}
$$

for $(f, g, h) \in \mathfrak{Y} \times \mathfrak{Y} \times \mathfrak{Y}$, that is, $T$ is a shrinking operator from $\mathfrak{Y} \times \mathfrak{Y} \times \mathfrak{Y}$ to $Y \times Y \times Y$.

Now, we show that $(U, V, W)$ is Hölder continuous. We will show that $\|U\|_{C^{0, \gamma}}$ is finite. Indeed,

$$
\begin{aligned}
U(x) & =\int_{\varepsilon}^{1}\left|G_{\alpha}^{\prime}(t)\right| \int_{B_{t}(x)} \frac{w^{r}(y) v^{q}(y)}{|y|^{\beta}} d y d t+\int_{1}^{\infty}\left|G_{\alpha}^{\prime}(t)\right| \int_{B_{t}(x)} \frac{w^{r}(y) v^{q}(y)}{|y|^{\beta}} d y d t \\
& =: U_{1}(x)+U_{2}(x) .
\end{aligned}
$$


For any $x, z \in \Omega$, we have

$$
\begin{aligned}
& \left|U_{1}(x)-U_{1}(z)\right| \\
& \quad=\left|\int_{\varepsilon}^{1}\right| G_{\alpha}^{\prime}(t)\left|\int_{B_{t}(x)} \frac{w^{r}(y) v^{q}(y)}{|y|^{\beta}} d y d t-\int_{\varepsilon}^{1}\right| G_{\alpha}^{\prime}(t)\left|\int_{B_{t}(z)} \frac{w^{r}(y) v^{q}(y)}{|y|^{\beta}} d y d t\right| \\
& \quad=\left|\int_{\varepsilon}^{1}\right| G_{\alpha}^{\prime}(t)\left|\left(\int_{B_{t}(x)} \frac{w^{r}(y) v^{q}(y)}{|y|^{\beta}} d y-\int_{B_{t}(z)} \frac{w^{r}(y) v^{q}(y)}{|y|^{\beta}} d y\right) d t\right| \\
& \quad \leq\|v\|_{\infty}^{q}\|w\|_{\infty}^{r} \int_{\varepsilon}^{1}\left|G_{\alpha}^{\prime}(t)\right|\left(\int_{\left(B_{t}(x) \backslash B_{t}(z)\right) \cup\left(B_{t}(z) \backslash B_{t}(x)\right)} \frac{1}{|y|^{\beta}} d y\right) d t .
\end{aligned}
$$

On the other hand, we get from [15]

$$
\int_{\left(B_{t}(x) \backslash B_{t}(z)\right) \cup\left(B_{t}(z) \backslash B_{t}(x)\right)} \frac{1}{|y|^{\beta}} d y \leq C t^{n-1-\beta}|x-z|^{\gamma} .
$$

Therefore,

$$
\left|U_{1}(x)-U_{1}(z)\right| \leq C\|v\|_{\infty}^{q}\|w\|_{\infty}^{r}|x-z|^{\gamma} \int_{\varepsilon}^{1}\left|G_{\alpha}^{\prime}(t)\right| t^{n-1-\beta} d t \leq C(\varepsilon)|x-z|^{\gamma}
$$

That is,

$$
\sup _{x \neq z} \frac{\left|U_{1}(x)-U_{1}(z)\right|}{|x-z|^{\gamma}} \leq C(\varepsilon)
$$

For the second part, we use a different approach. We have

$$
\begin{aligned}
U_{2}(x)-U_{2}(z) & \leq \int_{1}^{\infty}\left|G_{\alpha}^{\prime}(t)\right| \int_{B_{t(1+\delta)}(z)} \frac{w^{r}(y) v^{q}(y)}{|y|^{\beta}} d y d t-U_{2}(z) \\
& \leq \int_{1+\delta}^{\infty}\left|G_{\alpha}^{\prime}(t)\right| \int_{B_{t}(z)} \frac{w^{r}(y) v^{q}(y)}{|y|^{\beta}} d y d t(1+\delta)^{\alpha}-U_{2}(z) \\
& \leq U_{2}(z)\left[(1+\delta)^{\alpha}-1\right] \leq u(z)(1+\xi)^{\alpha-1} \delta \\
& \leq C\|u\|_{\infty} \delta \leq C\|u\|_{\infty}|x-z|^{\gamma} .
\end{aligned}
$$

Note that here we have applied the mean value theorem with $\xi$ valued between 0 and $\delta$. The same inequality holds for $U_{2}(z)-U_{2}(x)$. Therefore,

$$
\sup _{x \neq z} \frac{\left|U_{2}(x)-U_{2}(z)\right|}{|x-z|^{\gamma}} \leq C\|u\|_{\infty}
$$

By the definition of $U(x)$, we immediately have

$$
\|U\|_{\infty} \leq\|u\|_{\infty}
$$

Inequalities (4.2) and (4.3) imply that $U(x)$ is Hölder continuous, which together with (4.4) yields $U \in \mathfrak{X} \cap \mathfrak{Y}$. Similarly, we can show $V, W \in \mathfrak{X} \cap \mathfrak{Y}$. As a result, $(U, V, W) \in$ $(\mathfrak{X} \times \mathfrak{X} \times \mathfrak{X}) \cap(\mathfrak{Y} \times \mathfrak{Y} \times \mathfrak{Y})$. 
Finally, we show that $S_{\varepsilon}$ maps $(\mathfrak{X} \times \mathfrak{X} \times \mathfrak{X}) \cap(\mathfrak{Y} \times \mathfrak{Y} \times \mathfrak{Y})$ into itself. We only need to verify that if $(u, v, w) \in \mathfrak{X} \times \mathfrak{X} \times \mathfrak{X}$, then

$$
T_{\varepsilon}(u, v, w) \leq 2\|u\|_{\infty}+2\|v\|_{\infty}+2\|w\|_{\infty} .
$$

In fact,

$$
\begin{aligned}
T_{1}^{\varepsilon}(v, w)(x) & =\int_{0}^{\varepsilon}\left|G_{\alpha}^{\prime}(t)\right| \int_{B_{t}(x)} \frac{w^{r}(y) v^{q}(y)}{|y|^{\beta}} d y d t \\
& \leq\|v\|_{\infty}^{q}\|w\|_{\infty}^{r} \int_{0}^{\varepsilon}\left|G_{\alpha}^{\prime}(t)\right| \int_{B_{t}(x)} \frac{1}{|y|^{\beta}} d y d t \\
& \leq C\left(2\|u\|_{\infty}+2\|v\|_{\infty}+2\|w\|_{\infty}\right)^{q+r} \varepsilon^{\alpha-\beta} .
\end{aligned}
$$

Similarly,

$$
T_{2}^{\varepsilon}(w, u)(x) \leq C\left(2\|u\|_{\infty}+2\|v\|_{\infty}+2\|w\|_{\infty}\right)^{p+r} \varepsilon^{\alpha-\beta}
$$

and

$$
T_{3}^{\varepsilon}(u, v)(x) \leq C\left(2\|u\|_{\infty}+2\|v\|_{\infty}+2\|w\|_{\infty}\right)^{p+q} \varepsilon^{\alpha-\beta} .
$$

Choosing $\varepsilon$ sufficiently small, we obtain (4.5). The proof is complete.

\section{Competing interests}

The authors declare that they have no competing interests.

\section{Authors' contributions}

All authors contributed equally to the manuscript and typed, read, and approved the final manuscript.

\section{Acknowledgements}

The authors would like to thank Professor Jianfu Yang for his encouragement and useful suggestion. The first author was supported by NNSF of China (11261023, 11326092), Startup Foundation for Doctors of Jiangxi Normal University. The second author was supported by NNSF of China (11271170), the GAN PO 555 program of Jiangxi Province.

Received: 1 March 2014 Accepted: 24 May 2014 Published: 03 Jun 2014

\section{References}

1. Serrin, J: A symmetry problem in potential theory. Arch. Ration. Mech. Anal. 43, 304-318 (1971)

2. Gidas, B, Ni, WM, Nirenberg, L: Symmetry and related properties via the maximum principle. Commun. Math. Phys. 68, 209-243 (1979)

3. Caffarelli, L, Gidas, B, Spruck, J: Asymptotic symmetry and local behavior of semilinear elliptic equations with critical Sobolev growth. Commun. Pure Appl. Math. XLII, 271-297 (1989)

4. Gidas, B, Ni, WM, Nirenberg, L: Symmetry of positive solutions of nonlinear elliptic equations in $R^{n}$. In: Mathematical Analysis and Applications, Part A. Adv. Math. Suppl. Stud., vol. 7a, pp. 369-402. Academic Press, New York (1981)

5. Chen, WX, Li, CM, Ou, B: Classification of solutions for an integral equations. Commun. Pure Appl. Math. 59, 330-343 (2006)

6. Chen, WX, Li, CM, Ou, B: Classification of solutions for a system of integral equations. Commun. Partial Differ. Equ. 30, 59-65 (2005)

7. Chen, WX, Li, CM: Methods on Nonlinear Elliptic Equation. AIMS, Springfield (2010)

8. Jin, C, Li, CM: Symmetry of singular solutions of integral equations. Proc. Am. Math. Soc. 134, 1661-1670 (2006)

9. $\mathrm{Ma}, \mathrm{L}, \mathrm{Chen}, \mathrm{DZ}$ : Radial symmetry and monotonicity results for an integral equation. J. Math. Anal. Appl. 342, 943-949 (2008)

10. $\mathrm{Ma}, \mathrm{L}, \mathrm{Chen}, \mathrm{DZ}$ : Radial symmetry and uniqueness for positive solutions of a Schrödinger type system. Math. Comput. Model. 49, 379-385 (2009)

11. Lieb, E: Existence and uniqueness of the minimizing solution of Choquard's nonlinear equation. Stud. Appl. Math. 57, 93-105 (1977) 
12. $\mathrm{Ma}, \mathrm{L}, \mathrm{Zhao}, \mathrm{L}:$ Uniqueness of ground states of some coupled nonlinear Schrödinger systems and their application. J. Differ. Equ. 245, 2551-2565 (2008)

13. Lei, YT: On the regularity of positive solutions of a class of Choquard type equations. Math. Z. 273, 883-905 (2013)

14. Ma, F, Huang, X, Wang, L: A classification of positive solutions of some integral systems. Integral Equ. Oper. Theory 69 , 393-404 (2011)

15. Chen, XL, Yang, JF: Regularity and symmetry of positive solutions of an integral systems. Acta Math. Sci. 32B, 1759-1780 (2012)

16. Zhu, SR, Chen, XL, Yang, JF: Regularity, symmetry and uniqueness of positive solutions to a nonlinear elliptic system. Commun. Pure Appl. Anal. 13, 2685-2696 (2013)

10.1186/1029-242X-2014-222

Cite this article as: Chen and Wang: Symmetry and regularity of positive solutions to integral systems with Bessel potential. Journal of Inequalities and Applications 2014, 2014:222

Submit your manuscript to a SpringerOpen ${ }^{\circ}$ journal and benefit from:

- Convenient online submission

Rigorous peer review

- Immediate publication on acceptance

- Open access: articles freely available online

- High visibility within the field

- Retaining the copyright to your article

Submit your next manuscript at $>$ springeropen.com 